\title{
Development and characterization of novel auxetic structures based on re-entrant hexagon design produced from braided composites
}

\author{
P. Subramani ${ }^{\text {a }}$, Sohel Rana ${ }^{\text {a, * }}$, Bahman Ghiassi ${ }^{\text {b }}$, Raul Fangueiro ${ }^{\text {a }}$, Daniel V. Oliveira ${ }^{\text {b }}$, \\ Paulo B. Lourenco ${ }^{b}$, Jose Xavier ${ }^{c}$ \\ ${ }^{a}$ Center for Textile Science and Technology, University of Minho, Guimarães, Portugal \\ ${ }^{\mathrm{b}}$ ISISE, Department of Civil Engineering, University of Minho, Guimarães, Portugal \\ ${ }^{\mathrm{c}}$ INEGI, University of Porto, Porto, Portugal
}

\section{A R T I C L E I N F O}

\section{Article history:}

Received 3 November 2015

Received in revised form

13 January 2016

Accepted 24 February 2016

Available online 4 March 2016

\section{Keywords:}

A. Smart materials

B. Mechanical properties

C. Analytical modelling

D. Mechanical testing

E. Braiding

\begin{abstract}
A B S T R A C T
This paper reports the first attempt of developing macro-scale auxetic structures based on re-entrant hexagon design from braided composite materials for civil engineering applications. Braided composite rods (BCRs) were produced and arranged as longitudinal and horizontal elements to produce three types of auxetic structures: (1) basic re-entrant hexagon structure, (2) basic structure modified by adding straight longitudinal elements and (3): structure-2 modified by changing structural angle. The influence of various material and structural parameters as well as structure type on Poisson's ratio and tensile properties was thoroughly investigated. The auxetic behaviour was found to strongly depend on the structural angle and straight elements, resulting in lower auxeticity with lower angles and in presence of straight elements. Material parameters influenced the auxetic behaviour to a lesser extent and a decrease in auxetic behaviour was noticed with increase in core fibre linear density and using stiffer fibres such as carbon. The reverse effect was observed in case of tensile strength and work of rupture. Among these structures, structure- 3 exhibited good auxetic behaviour, balanced tensile properties, and high energy absorption capacity and their auxetic behaviour could be well predicted with the developed analytical model. Therefore, these novel structures present good potential for strengthening of civil structures.
\end{abstract}

๑) 2016 Elsevier Ltd. All rights reserved.

\section{Introduction}

Advanced fibre reinforced polymer (FRP) composites find numerous applications in automobile, marine and aerospace industries due to their enhanced engineering properties such as low density, high specific strength and stiffness, high damping characteristics, high fatigue endurance and low thermal co-efficient (in fibre direction), etc. [1-4]. Recently, composites have been applied widely in civil engineering applications to replace conventional materials (concrete and steel) or ceramic based composites [1]. Composites are introduced into structural elements to improve their flexural resistance, shear strength, confinement, bending property, etc. [1]. Nowadays, research is being carried out to use composite materials in structural elements to improve their resistance against earthquake, blast or impact loads caused by

\footnotetext{
* Corresponding author. Center for Textile Science and Technology, University of Minho, Guimaraes, Azurem Campus 4800-058, Portugal. Tel.: +351 910623763.

E-mail address: sohel.rana@det.uminho.pt (S. Rana).
}

explosions [5]. Capacity to absorb energy is one of the primary requirements for these applications and, therefore, designing strengthening materials possessing excellent energy absorption capability is highly demanding for these structural applications.

Braided composites rods (BCRs) have been introduced in the civil structural applications for the replacement of steel which presents serious corrosion problems. Braiding technology is a low cost and well established technology in which intertwining of three or more strands of yarns forms tubular fabric structures with different combinations of linear or twisted core (axial) materials. Impregnation of axial fibres with polymeric resin before introducing into the braided structure and subsequent curing forms composite materials with braided architecture or BCR. The loaddeformation behaviour of braided structures of composites can be tailored by choosing suitable fibres for the sheath and core components. The most commonly used core fibres for civil applications are glass, basalt and carbon fibres. BCRs offer several advantages over the other types of FRP rods such as simple and economical manufacturing process, tailorable mechanical properties and good 
bonding behaviour with cementitious matrices [6-8]. BCRs have been already demonstrated to possess high potential for application in concrete reinforcement and monitoring $[9,10]$, masonry wall strengthening [11] and reinforcement of soils for geotechnical applications [12]. BCRs were used in these applications in the form of meshes made of longitudinal and transverse elements and currently, efforts are directed towards arranging these elements in especial structural designs (such as auxetic), in order to greatly enhance the energy absorption capacity.

Auxetic materials are special kind of innovative materials which exhibit negative Poisson's ratio. Unlike conventional materials, auxetic materials expand in transverse direction in tension and shrink laterally in compression [13,14]. Auxetic materials are gaining considerable interest over the past three decades due to their superior physical properties such as improved strength, enhanced fracture resistance, superior energy absorption, superior acoustic behaviour, good indentation resistance, and improved damping [15-22]. Auxetic materials are available in different forms starting from micro to nano scale such as liquid crystalline polymers, microporous polymer, fibres, foams, honeycombs, biomaterials, nano composites, FRP composites, etc. Auxetic composites can find potential applications in automotive, protection and aerospace industry, where non-auxetic composites with high specific strength and stiffness are currently used [13]. The auxetic property can also be achieved with certain structural designs. In the last few decades, dissimilar geometric structures and models exhibiting auxetic behaviour like 2D and 3D re-entrant structures, rotating rigid/semi-rigid units, chiral structures, etc. have been proposed, studied and tested for their mechanical properties [14,17,23-26].

Among many auxetic structural designs, re-entrant hexagon or bow-tie or butterfly design [27] has been investigated frequently by several researchers. Re-entrant is the internal geometry of many structures, which are observed in macro, micro and nano forms [28]. As of now, re-entrant design was adopted to produce various auxetic materials such as foam cores of sandwich panel for aerospace and air filtration applications [24,29,30], flat and warp knitted fabrics [28,31], molecular auxetic, e.g. (n,m) - reflexyne [19,32], cellular tubes for angioplasty or Annuloplasty rings [33], honeycomb core from Kevlar/epoxy and recycled rubber for sandwich panels [34,35], etc.

Till date, development of auxetic structures at macro-scale and for strengthening of structural elements has been rarely reported. Recently, the authors developed, for the first time, auxetic structures from BCRs based on missing rib or lozenge grid and characterized their auxetic and tensile behaviours [36]. Although the developed structures exhibited negative Poisson's ratio, the structural parameter (opening between the meshes) and mechanical property (mainly stiffness at small strains) did not fit very well with the requirements of structural applications. Motivated by this initial study and in searching for a better structural design, attempt has been made in the present research to develop new types of auxetic structures from BCR based on re-entrant hexagon design. According to the author's knowledge, this type of auxetic structures has been developed for the first time in macro-scale and using braided composite materials. For the civil engineering applications, braided composites can be advantageous due to their especial textured surface providing excellent bonding characteristics with cementitious materials [11]. The developed auxetic structures were characterized for their auxetic and tensile behaviours and the influence of structure type and structural (angle) as well as material parameters (type of fibre and linear density) was thoroughly studied. In addition, analytical models have been developed and the experimental results have been compared with the analytical results.

\section{Materials and methods}

\subsection{Materials}

For the production of composite rods, glass fibre roving with linear density of 1200 tex and 4800 tex was purchased from Owens Corning, France. For the same purpose, basalt fibre roving with linear density of 4800 tex and carbon fibre roving with linear density of 1600 tex were purchased from Basaltex, Belgium and Toho Tenax, Germany, respectively. The epoxy resin used to coat the structures was supplied by Sika, Germany, in two components: Biresin CR83 Resin and Biresin CH-83-2 Hardener. The resin and hardener components were mixed in a weight ratio of 100:30 prior to application. The important characteristics of fibre and resin system are listed in Table 1.

\subsection{Fabrication of braided composite rods and auxetic structures}

Triaxial braided structures were produced in a vertical braiding machine using polyester multi-filament yarns (with linear density of 110 tex) in the sheath and glass/basalt/carbon multi-filament rovings as the core material. During the braiding process, sixteen polyester filament bobbins were used to supply the sheath yarns, which were then braided around the core fibres [6-8]. Produced braided structures were next used to develop three types of auxetic structures, as shown in Fig. 1. The first structure (structure-1) was developed using the basic re-entrant honeycomb design. In structure-2, the basic design was modified with straight longitudinal rods. Structure-2 was further modified to improve the tensile behaviour using higher angle of longitudinal rods, resulting in structure-3. For each type, three samples were produced keeping the total length and width as $40 \mathrm{~cm}$ and $11 \mathrm{~cm}$, respectively, with additional length for clamping during tensile testing. Following steps were performed to produce the auxetic structures: the auxetic structural design (Fig. 1) was drawn on a white chart paper; (2) the chart paper was placed on a board and the braided structures were placed over the drawn design firmly with help of adhesive tape; (3) the joints were tied by polyester filaments and epoxy resin was applied over the structures using a brush; (4) after curing, the structures were removed from the board. The braided structures after resin application and curing became circular composites termed as braided composite rods (BCR). The weight percentage of core fibre in each of these rods was around $51 \pm 2 \%$. Resin application was necessary to give sufficient mechanical stability to the braided materials in order to handle them easily and turn them in to auxetic structures. In absence of resin, there may be slippage between the core and sheath as well as between the core fibres causing poor mechanical properties.

\subsection{Parameters of developed structures}

In order to study the influence of different parameters, auxetic structures were produced using different types of core fibre having

Table 1

Physical properties of core fibres and resin.

\begin{tabular}{llllll}
\hline S. No. & Properties & Basalt & Glass & Carbon & Epoxy \\
\hline 1 & Density $\left(\mathrm{g} / \mathrm{cm}^{3}\right)$ & 2.63 & 2.62 & 1.77 & 1.15 \\
2 & Filament diameter $(\mu \mathrm{m})$ & 17 & - & 13 & - \\
3 & Tensile strength $(\mathrm{MPa})$ & $>4000$ & $3100-3800$ & 4400 & 122 \\
4 & Tensile modulus $(\mathrm{GPa})$ & 87 & $80-81$ & 240 & 3.3 \\
5 & Elongation $(\%)$ & - & - & 1.8 & 6.7 \\
\hline
\end{tabular}




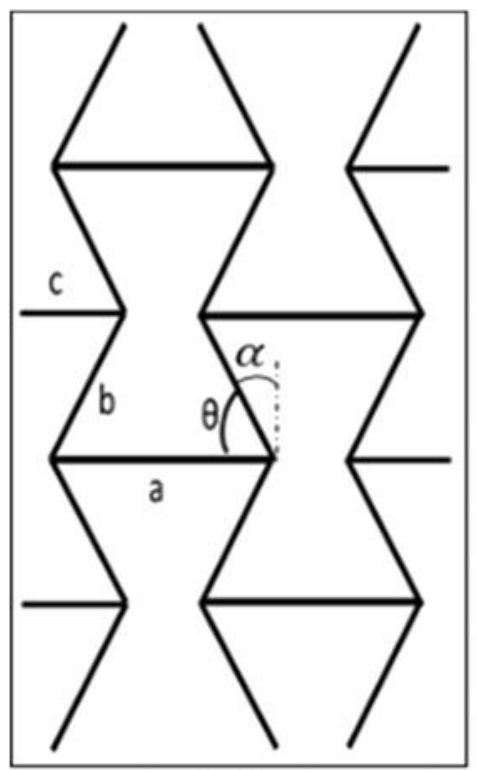

(a)

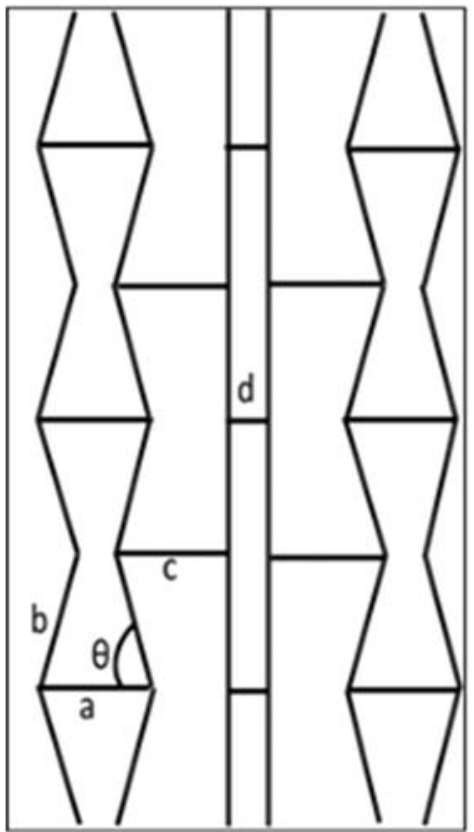

(d)

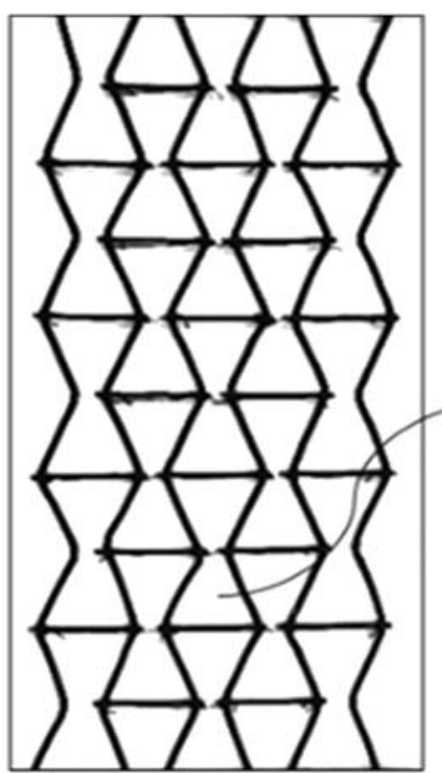

(b)

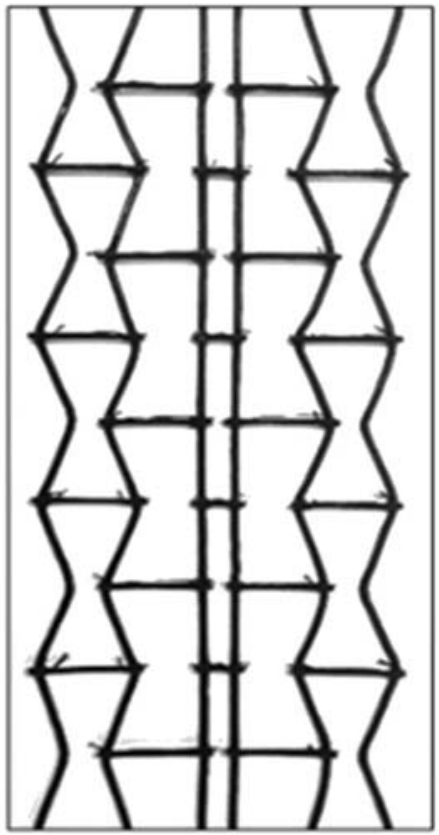

(e)

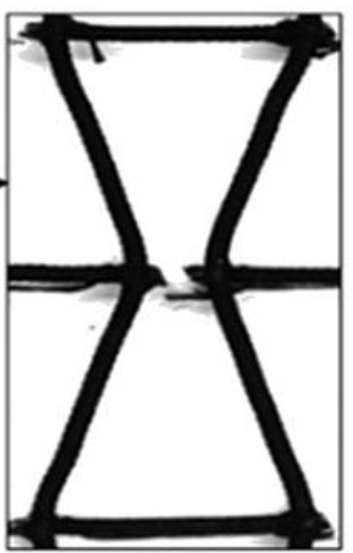

(c)

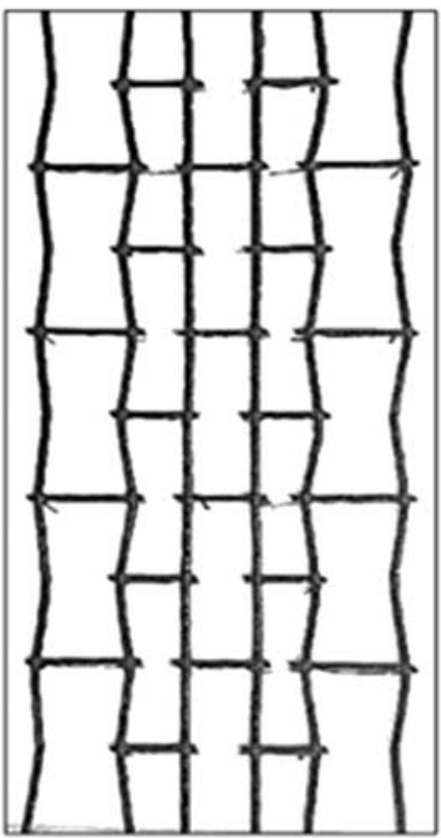

(f)

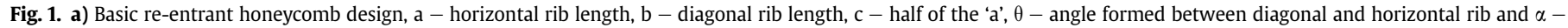

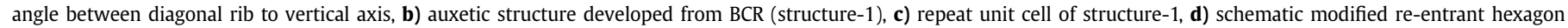
design, e) structure-2, and f) structure-3.

different linear densities (2400 tex, 4800 tex and 6000 tex glass fibre; 4800 tex basalt fibre and 4800 tex carbon fibre). Moreover, modification of basic structures through addition of straight longitudinal rods and change in structural angle resulted in different structural parameters, which are listed in Table 2 along with material parameters.

\subsection{Measurement of Poisson's ratio and tensile properties}

The measurement of Poisson's ratio and tensile properties of the developed auxetic structures were carried out in a Universal Tensile
Testing Machine. The cross-head speed was kept at $25 \mathrm{~mm} / \mathrm{min}$. White marks were painted on the structures at top, middle and bottom, as shown in Fig. 2. During tensile testing, the video of sample deformation with load was captured using Canon EOS 650D and later, the video was converted in to images at specific intervals (per second) using an image analysis software (Image J). The distance between the marks in the structures, both in longitudinal and transverse directions, was measured in pixels using Image software. The longitudinal and transverse strains were then calculated by using following formulae: 
Table 2

Material and structural parameters of developed auxetic structures.

\begin{tabular}{|c|c|c|c|c|c|c|}
\hline Structure & Core fibres & Core fibre linear density, tex & Angle & Value (degrees) & Rib & Length $(\mathrm{cm})$ \\
\hline \multirow[t]{3}{*}{1} & Glass & $2400,4800,6000$ & $\theta$ & 76 & $\mathrm{a}$ & 3.0 \\
\hline & Basalt & 4800 & & & $\mathrm{~b}$ & 4.1 \\
\hline & Carbon & 4800 & & & & \\
\hline \multirow[t]{4}{*}{2} & Glass & 4800 & $\theta$ & 76 & $\mathrm{a}$ & 3.0 \\
\hline & & & & & $\mathrm{b}$ & 4.1 \\
\hline & & & & & c & 3.0 \\
\hline & & & & & d & 1.0 \\
\hline \multirow[t]{4}{*}{3} & Glass & 4800 & $\theta$ & 83 & $\mathrm{a}$ & 3.0 \\
\hline & & & & & b & 4.0 \\
\hline & & & & & c & 2.0 \\
\hline & & & & & d & 2.0 \\
\hline
\end{tabular}

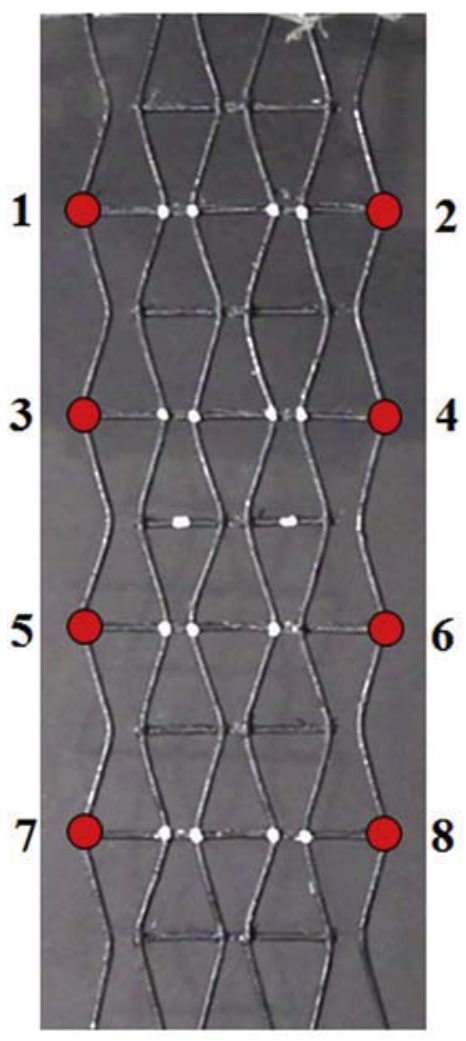

(a)

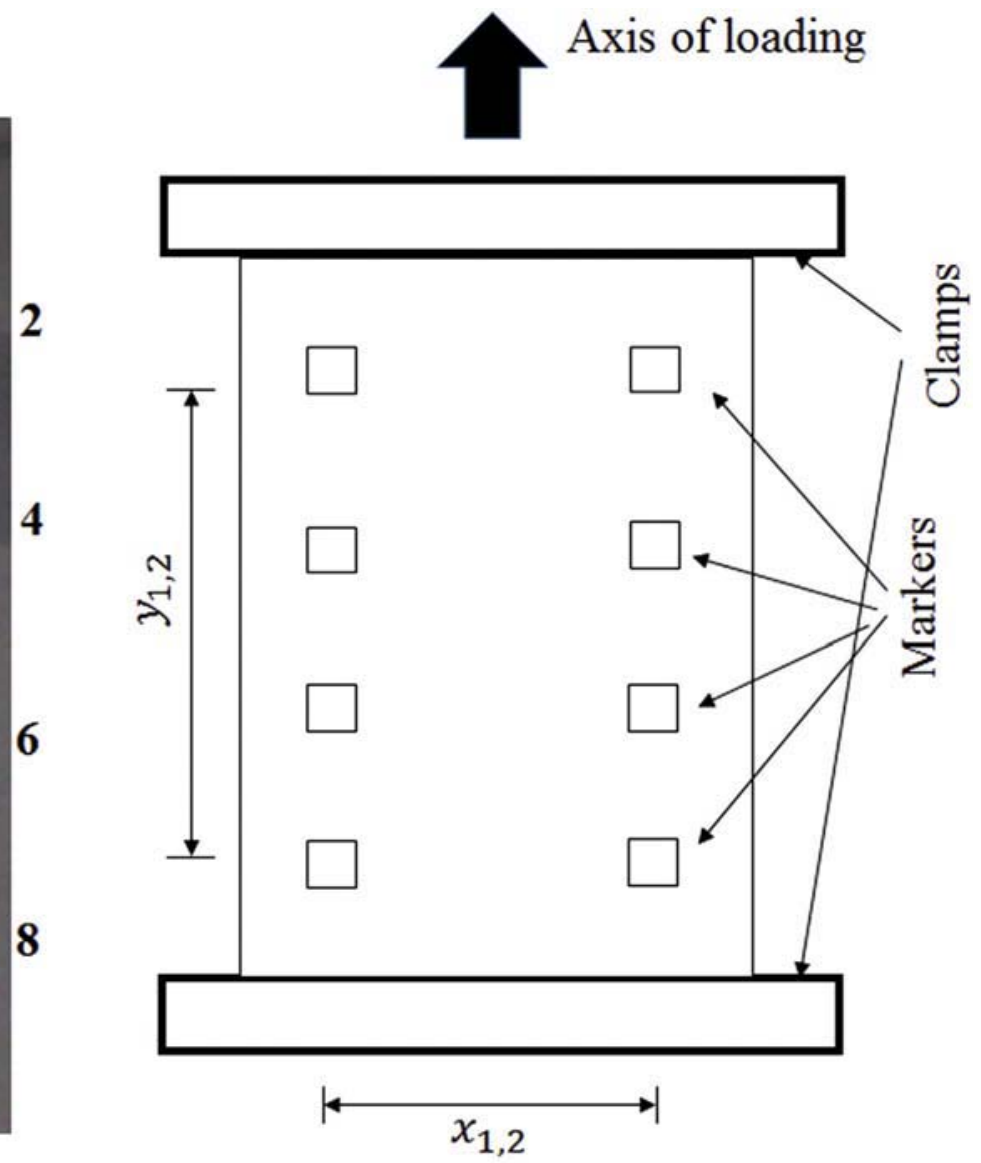

(b)

Fig. 2. a) Auxetic structure with painted marks, and b) schematic points for strain calculation.

$\varepsilon_{x}=\frac{x_{n}-x_{0}}{x_{0}}$

$\varepsilon_{y}=\frac{y_{n}-y_{0}}{y_{0}}$

where $x_{n}$ and $y_{n}$ are the distance between the points at nth loading, $x_{0}$ and $y_{0}$ are the original distance between the marks at zero loading. The average transverse strain was calculated by averaging the lateral strains calculated at the middle points $(3,4$ and 5,6$)$. Similarly, the average longitudinal strain was calculated from longitudinal strains measured from the left and right points (1, 7 and 2, 8 ) of the structures [25]. The measurement principle has been illustrated in Fig. 2b. Later, Poisson's ratio was calculated from the average strains using Eq. (3):

$\nu_{x y}=-\frac{\left\langle\varepsilon_{x}\right\rangle}{\left\langle\varepsilon_{y}\right\rangle}$ 


\section{Results and discussion}

\subsection{Auxetic behaviour of basic structures}

The developed structures based on re-entrant hexagon design exhibits negative Poisson's ratio. When tensile load is applied to the structure in longitudinal direction, the angle of longitudinal composite rods become progressively higher, resulting in continuous straightening of these rods until angle $\theta$ becomes $90^{\circ}$. Straightening of longitudinal rods induces outward lateral movements in the transverse rods, resulting in transverse expansion of the structures and auxetic behaviour. The maximum transverse deformation in the structures is achieved when the longitudinal rods become fully straight and further loading causes extension and finally breakage of the longitudinal rods. Therefore, under tensile loading, the longitudinal rods initially undergo straightening and subsequently, tensile extension and breakage and the transverse expansion of the structures mainly occurs during straightening of the longitudinal elements. To illustrate this point, the deformation of a repeat unit cell at various stages during tensile testing is presented in Fig. 3.

\subsection{Effect of core fibre on auxetic behaviour}

In order to investigate the influence of core fibre type, auxetic structures were produced with braided rods consisting of glass, basalt and carbon core fibres with same linear density, 4800 tex. As shown in Fig. 4 and listed in Table 3, the core fibre type exhibit significant influence on auxetic behaviour and the trend of Poisson's ratio change with longitudinal strain is same for all the fibres. Poisson's ratio first increases with strain and then decreases with further strain increase.

The decrease in Poisson's ratio after certain strain level is attributed to the restriction in the free transverse movement of the structures by the clamping systems present at top and bottom of

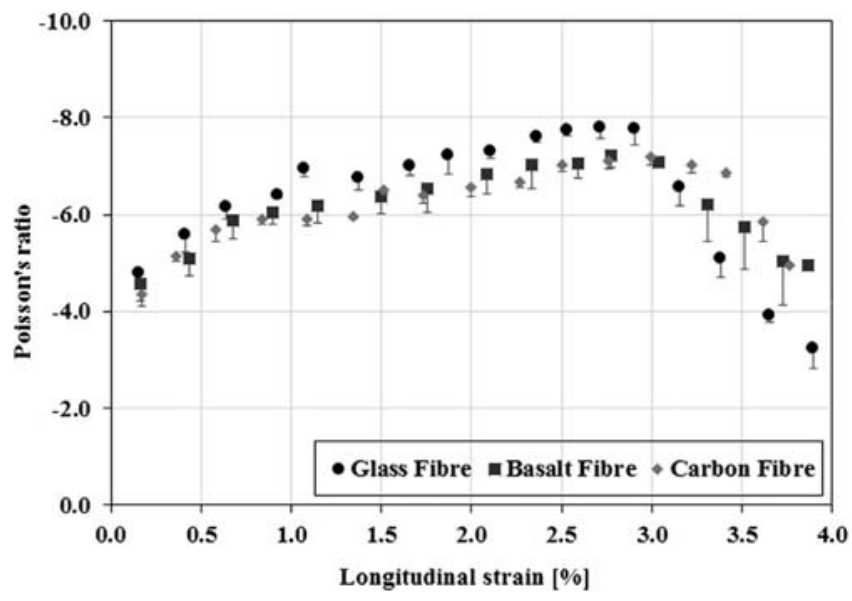

Fig. 4. Auxetic behaviour of structure-1 containing different types of core fibres (linear density 4800 tex).

the structures. This results in first bending and subsequently breakage of the transverse rods prior to the longitudinal rods' failure. The highest auxetic behaviour is noticed for glass fibre structure, followed by basalt and carbon fibre structures. Maximum Poisson's ratio obtained with glass fibre was $~ 8 \%$ and $~ 11 \%$ higher as compared to basalt and carbon fibre based structures, respectively. This difference in auxetic behaviour of the structures is related to their stiffness. The stiffer fibre based structures (carbon) show more resistance to deformation and therefore, results in lower Poison's ratio. Therefore, the developed structures shows Poisson's ratio in the following order, which is reverse to the stiffness of core fibres: Poisson's ratio glass structure $>$ Poisson's ratio basalt structure $>$ Poisson's ratio carbon structure.

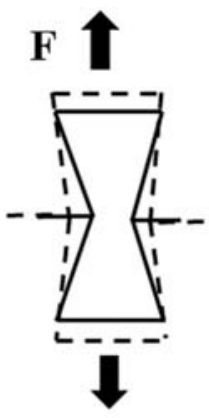

(a)

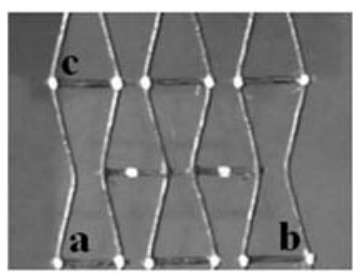

Distance $a-b=11.09 \mathrm{~cm}$ $\mathrm{a}-\mathrm{c}=7.95 \mathrm{~cm}$

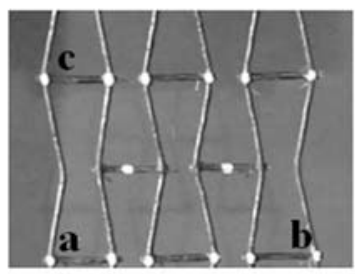

Distance $a-b=11.68 \mathrm{~cm}$ $a-c=8.00 \mathrm{~cm}$

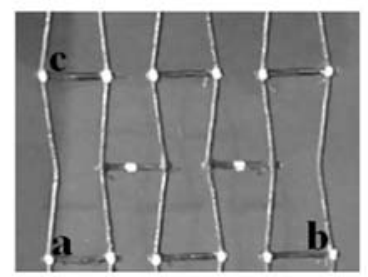

Distance

$a-b=12.46 \mathrm{~cm}$

$a-c=8.08 \mathrm{~cm}$

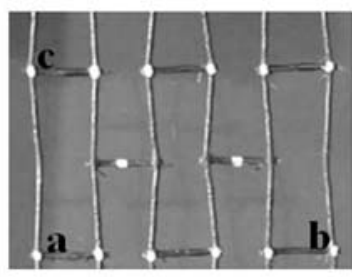

Distance $a-b=12.99 \mathrm{~cm}$ $\mathrm{a}-\mathrm{c}=8.12 \mathrm{~cm}$

(b)

Fig. 3. a) Schematic of unit cell with force and movement and b) deformation of repeat unit cell of basic auxetic structure at different stages during tensile loading. 
Table 3

Poisson's ratio of auxetic structures produced from glass, basalt and carbon fibres.

\begin{tabular}{|c|c|c|c|c|}
\hline Core fibre (tex) & Type of fibre & Average diameter of BCR ( $\mathrm{mm})$ & Average Max. Poisson's ratio & Percentage of change in Poisson's ratio w.r.t glass \\
\hline 4800 & Glass & $2.39(2.08)$ & $-8.04(3.29)$ & - \\
\hline 4800 & Basalt & $2.39(3.24)$ & $-7.37(6.32)$ & $\downarrow 8.3$ \\
\hline 4800 & Carbon & $2.91(4.00)$ & $-7.20(4.17)$ & 10.4 \\
\hline
\end{tabular}

Note: the values in the bracket are $\mathrm{CV} \%$.

\subsection{Effect of linear density of braided composite rods on auxetic behaviour}

The influence of linear density of core fibres on Poisson's ratio of developed structures can be noticed from Table 4 and Fig. 5. It is also clear from Table 4 that the diameter of BCRs increases with increasing linear density of core fibre. An increase in linear density of core fibres results in decrease in the auxetic behaviour of developed structures. A decrease in negative Poisson's ratio by $17 \%$ is noticed by increasing glass core linear density from 2400 tex to 6000 tex. This is attributed to the fact that higher diameter (i.e. high linear density core) horizontal and vertical elements present more resistance towards deformation resulting in lower structural movement and Poisson's ratio.

\subsection{Effect of structural angle on auxetic behaviour}

Table 5 shows the influence of structural angle $\theta$ on Poisson's ratio of structure-1. It should be noted that $\theta$ has been measured from the same structure during tensile deformation at different strain levels. It can be seen that an increase in $\theta$ by keeping other structural parameters ' $a$ ' and ' $b$ ' constant, Poisson's ratio increases. This is due to the fact that when the angle of longitudinal rods increases, they take higher axial loads as compared to the rods with lower angles, leading to higher structural deformation and Poisson's ratio. By changing $\theta$ from 74.5 to 85.0, Poisson's ratio increases by $54 \%$. Therefore, it is possible to obtain auxetic structures with different Poisson's ratio through easy adjustment of their structural angles.

\subsection{Influence of structural modification on auxetic behaviour}

The re-entrant auxetic structure, i.e. the basic structure was modified with straight longitudinal rods to improve their tensile behaviour, so that these auxetic structures will be suitable for strengthening of structural elements. The auxetic behaviour of the modified structure is shown in Fig. 6. The results show that auxetic behaviour of structure-2 (Fig. 6a) decreases as compared to the basic structure and the maximum achievable Poisson's ratio is -3.76 . The overall decrease in the Poisson's ratio of structure- 2 is attributed to the resistance of the straight rods towards deformation of structures. The trend of Poisson's ratio change with tensile strain is, however, very similar to the basic structure, except a strain

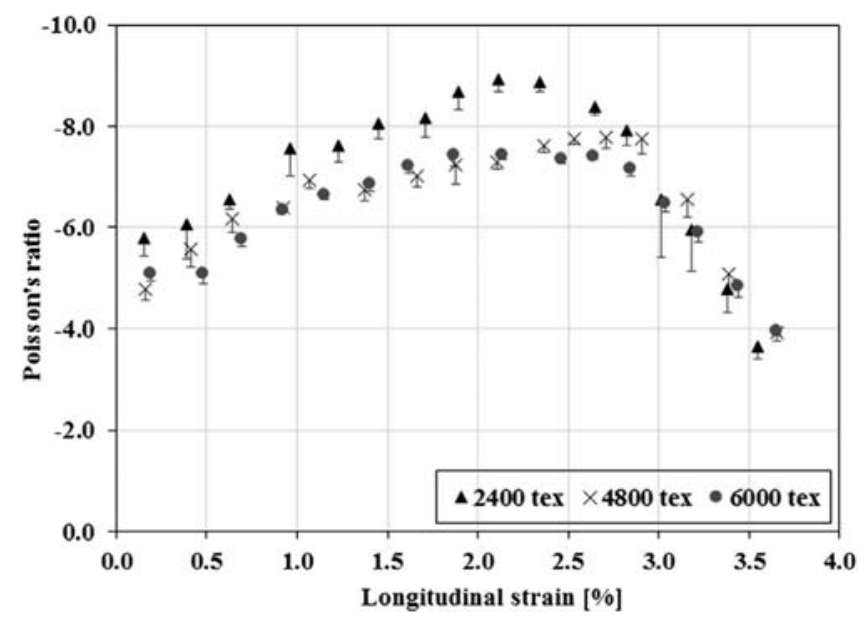

Fig. 5. Auxetic behaviour of structure-1 having different linear density of glass fibre roving as core.

region (indicated in the graph by arrow) in which Poisson's ratio decreases with strain. This region is due to the breakage of straight longitudinal rods, after which load is completely transferred to the diagonal longitudinal rods and the structure behaves again in the similar way to the basic structure. However, structure-3, in which the angle was further increased besides incorporating straight longitudinal rods, shows similar behaviour to the basic structure. The region in which Poisson's ratio decreases with strain, as observed in case of structure-2, is absent due to well balance of load sharing by the straight and diagonal longitudinal rods and smooth transfer of load, once the straight rods break. Poisson's ratio is also higher in this case due to higher structural angle, as shown in Fig. 6b, reaching maximum Poisson's ratio of -5.93 .

\section{Analytical model}

\subsection{Analytical model for Structure-1: calculation of strain and} Poisson's ratio

The re-entrant auxetic structure (Fig. 1b) will expand transversely while load applied in longitudinal direction. This is due to fact that diagonal longitudinal ribs or elements will move to the

Table 4

Poisson's ratio of auxetic structures produced from glass fibres with different linear densities.

\begin{tabular}{|c|c|c|c|}
\hline Glass fibre (Tex) & Average diameter of $\mathrm{BCR}(\mathrm{mm})$ & Average Max. Poisson's ratio & Percentage of change in Poisson's ratio w.r.t 2400 tex \\
\hline 2400 & $2.06(3.90)$ & $-9.00(4.24)$ & - \\
\hline 4800 & $2.39(2.08)$ & $-8.04(3.29)$ & $\downarrow \quad 10.7$ \\
\hline 6000 & $2.73(2.45)$ & $-7.47(3.03)$ & $\downarrow \quad 17.0$ \\
\hline
\end{tabular}

Note: the values in the bracket are CV\%. 
Table 5

Structural angle and Poisson's ratio of structure-1 at various stages of loading.

\begin{tabular}{|c|c|c|c|c|c|}
\hline \multirow[t]{2}{*}{ Stage } & \multirow[t]{2}{*}{ Angle $\theta$} & \multicolumn{4}{|l|}{ Experimental } \\
\hline & & Transverse strain, \% & Longitudinal strain, \% & Poisson's ratio & Percentage increase in Poisson's ratio w.r.t stage 1 \\
\hline 1 & 74.5 & 1.03 & 0.22 & -4.57 & \\
\hline 2 & 77.0 & 7.41 & 1.58 & -4.70 & 2.8 \\
\hline 3 & 80.0 & 16.53 & 2.63 & -6.28 & 个 37.4 \\
\hline 4 & 81.5 & 19.22 & 2.89 & -6.64 & 个 45.3 \\
\hline 5 & 83.0 & 24.94 & 3.69 & -6.70 & 个 46.6 \\
\hline 6 & 85.0 & 29.66 & 4.21 & -7.05 & 4 54.3 \\
\hline
\end{tabular}

$\mathrm{a}-2.89 \mathrm{~cm}$ and $\mathrm{b}-3.98 \mathrm{~cm}$.

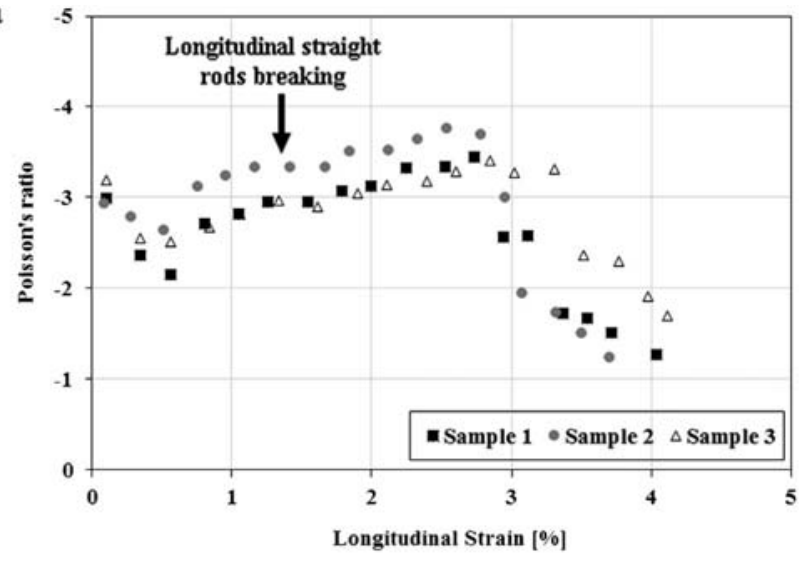

b

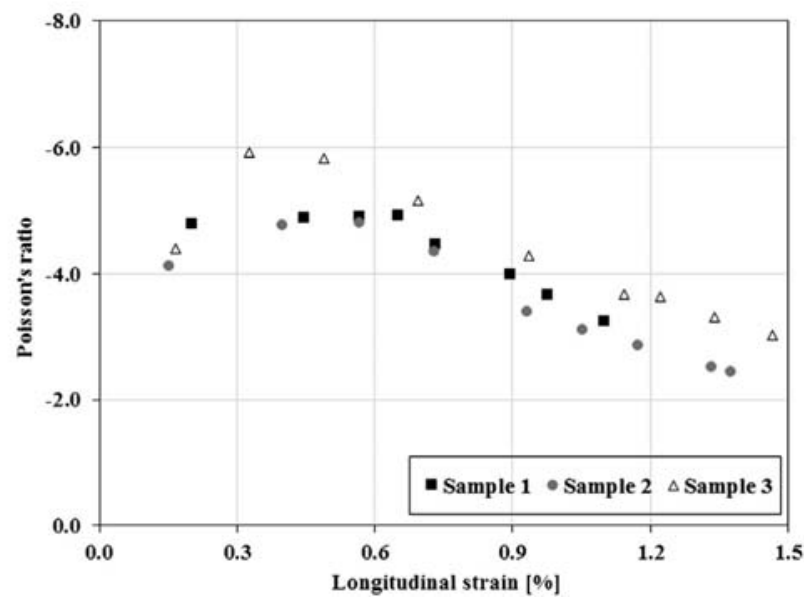

Fig. 6. a) Auxetic behaviour of structure-2 made of glass fibre BCRs, b) Auxetic behaviour of structure-3 made of glass fibre BCRs.

vertical disposition, which results an increase in the distance between the diagonal elements through the connection of horizontal elements. Consequently, the auxetic effect is achieved. Based on the geometrical analysis, Poisson's ratio $(v)$, axial strain $\left(\varepsilon_{a}\right)$ and transverse strain of the re-entrant auxetic structure formed with rigid rods can be theoretically calculated by following formulae's [28],

$\nu=\frac{b \sin \theta_{0}\left(\cos \theta_{0}-\cos \theta\right)}{\left(a-b \cos \theta_{0}\right)\left(\sin \theta_{0}-\sin \theta\right)}$ $\varepsilon_{a}=\frac{\sin \theta}{\sin \theta_{0}}-1$

$\varepsilon_{T}=\frac{b\left(\cos \theta_{0}-\cos \theta\right)}{\left(a-b \cos \theta_{0}\right)}$

The auxetic behaviour of structure- 1 has been calculated using Eq. (4) and Eq. (5) and compared with experimental values in Fig. 7. The parameters $\mathrm{a}, \mathrm{b}$ and angle $\theta$ which are required for analytical calculations are measured from images taken during testing at different stages. Poisson's ratio values are calculated until the structures deform transversely. The results show that the analytical Poisson's ratio is larger compared to experimental Poisson's ratio but the trend of its changes are similar. This can be due to different factors: (a) clamping method during the tests: the analytical method considers free deformation in the transverse direction, whereas during experimental evaluation, clamping systems at top and bottom do not allow free movement of the structures in the transverse direction. Although the experimental calculation of the Poisson's ratio is performed in the middle of the structure, there still can be some boundary conditions effects; (b) hindrance to the free movement at the joints: as the undulated elements of the auxetic structures were produced by bending a continuous rod, the joints of the bent elements are truly not the hinging points, where complete free rotation of the elements are possible. Hindrance to the free movement at these points may also reduce the transverse deformation and experimental Poisson's ratio; and (c) assumptions considered in the developed analytical model: the analytical

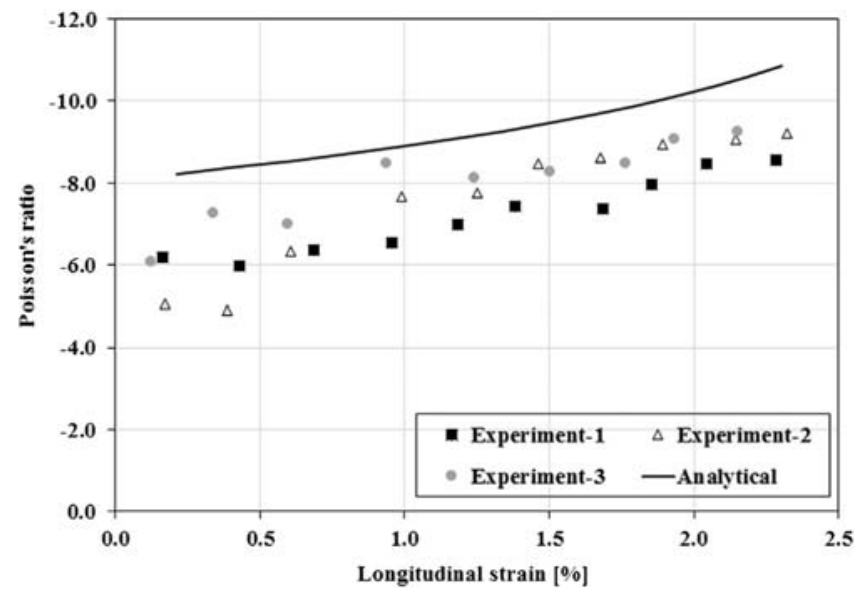

Fig. 7. Poisson's ratio of auxetic structure-1: analytical vs. experimental results. 


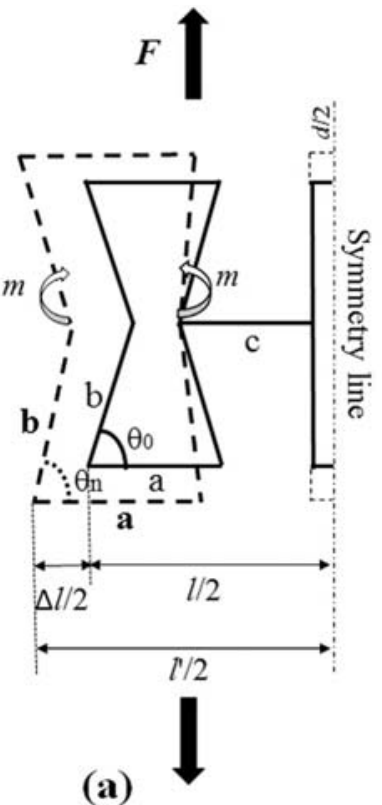

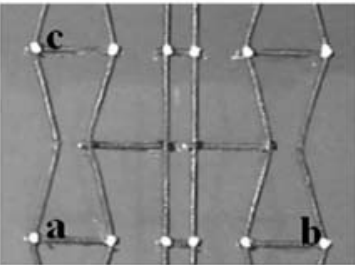

Distance $a-b=11.06 \mathrm{~cm}$ $\mathrm{a}-\mathrm{c}=7.99 \mathrm{~cm}$

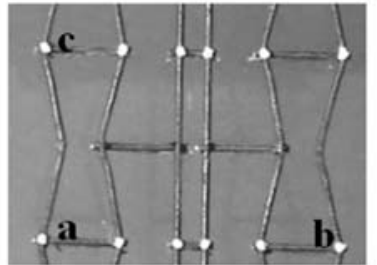

Distance

$a-b=11.38 \mathrm{~cm}$ $a-c=8.06 \mathrm{~cm}$

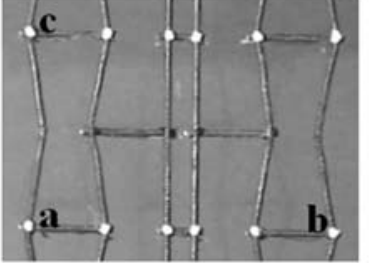

Distance

$a-b=11.68 \mathrm{~cm}$

$\mathrm{a}-\mathrm{c}=8.13 \mathrm{~cm}$

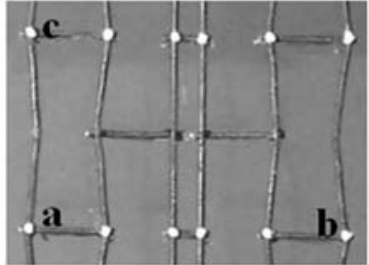

Distance

$a-b=12.06 \mathrm{~cm}$

$\mathrm{a}-\mathrm{c}=8.20 \mathrm{~cm}$

(b)

Fig. 8. a) Unit cell of structure-2, b) load and momentum act on the unit cell while axial loading, and c) unit cell displacement during tensile loading.

formulae are developed based on the assumption that only hinging mechanisms at the joints are governing the deformation of the structure. This means that it is assumed that the ribs are rigid, they do not change in length or bend, and they can rotate freely at the rib bases. Even though BCRs are rigid, there may be always small flexural deformation, which is not taken into account in the analytical model. On the other hand, the ribs may also stretch in large longitudinal strains which is also not taken into account. If these deformations are considered in the analytical model, it will provide larger longitudinal strains at the same transverse strains and thus smaller Poisson's ratio.

\subsection{Analytical model for Structure-2: calculation of strains and Poisson's ratio}

To study the auxetic behaviour of structure-2 analytically, the same equation, i.e. Eq. (5) can be used to calculate the longitudinal strains as a function of angle, $\theta$. However, Eq. (6) needs some revision as the unit cell is different in this structure. According to the experimental observations, the deformational mode of the structure is presented in Fig. 8. Assuming that the whole tested structure is a unit cell, the transverse strain can be written as presented in Eq. (7). The width of the structure (unit cell) is taken equal to $l$. Having the change of the unit cell width in each load level, $\Delta l$, the transverse strain can be obtained as $\varepsilon_{T}=\frac{\Delta l}{l}$. The deformed shape and free body diagram of half of the unit cell (due to its symmetry) is presented in Fig. 8. According to this diagram, the width can be obtained as $\frac{L}{2}=\frac{d}{2}+c+(a-b \cos \theta)$. The changes in the width with the changes of $\theta$ can be obtained as

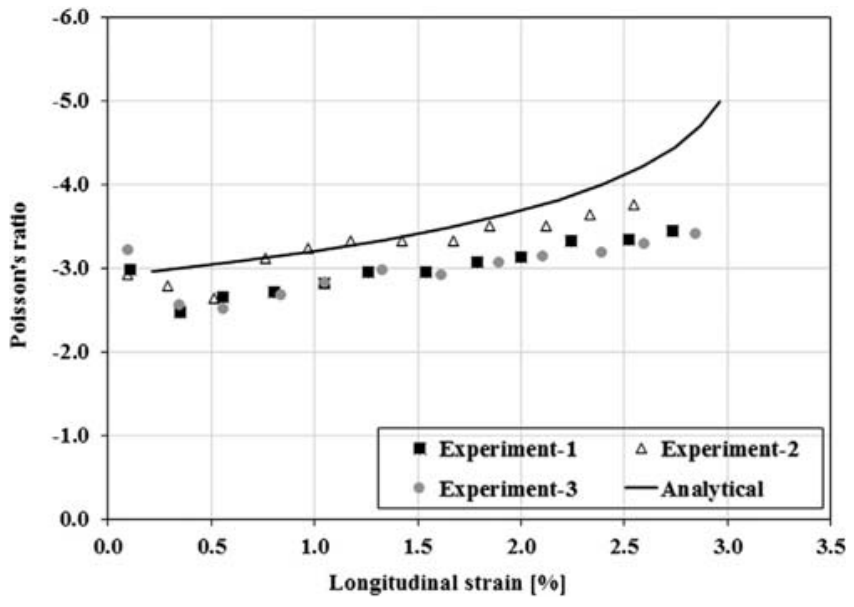

Fig. 9. Poisson's ratio of the structure-2: analytical vs. experimental results. 
Table 6

Tensile properties of various developed auxetic structures.

\begin{tabular}{|c|c|c|c|c|c|}
\hline Structure & Fibre type & Tex & Avg. Max. Tensile load, kN & Avg. Elongation at max. Tensile load, \% & Avg. Work of rupture, $\mathrm{J}$ \\
\hline$S-1$ & Glass & 2400 & $9.21(0.46)$ & $5.72(2.10)$ & $93.31(4.82)$ \\
\hline$S-1$ & Glass & 4800 & $10.40(8.33)$ & $5.14(5.45)$ & $98.20(9.40)$ \\
\hline$S-1$ & Glass & 6000 & $15.43(8.33)$ & $4.82(9.63)$ & $139.40(11.82)$ \\
\hline$S-1$ & Basalt & 4800 & $14.58(9.85)$ & $5.92(10.88)$ & $147.00(8.85)$ \\
\hline$S-1$ & Carbon & 4800 & $22.25(12.08)$ & $6.20(12.49)$ & $257.20(3.68)$ \\
\hline$S-2$ & Glass & 4800 & $8.23(7.31)$ & $4.90(6.57)$ & $93.7(4.96)$ \\
\hline$S-3$ & Glass & 4800 & $15.23(0.88)$ & $4.04(12.15)$ & $150.9(9.00)$ \\
\hline
\end{tabular}

$\Delta l / 2=b\left(\operatorname{Cos} \theta-\operatorname{Cos} \theta_{0}\right)$ leading to the following relation for the transverse strain:

$\varepsilon_{T}=\frac{\Delta l}{l}=\frac{2 b\left[\operatorname{Cos} \theta n-\operatorname{Cos} \theta_{0}\right]}{l}$

Using the longitudinal and transverse strains, the structural Poisson's ratio can be obtained. Poisson's ratio of this structure calculated by analytical and experimental methods are shown in the Fig. 9. The result shows that Poisson's ratio calculated analytically is almost similar to experimental values. It is found that the Poisson's ratio of Structure- 2 can be predicted using given analytical formulae.

\section{Tensile properties of auxetic structures}

\subsection{Tensile behaviour of developed auxetic structures}

The tensile properties of different auxetic structures are listed Table 6. It can be noticed that the maximum tensile load is the highest for carbon, followed by basalt and glass. The tensile load bearing capacity of these structures is strongly dependent on the core fibre's tensile properties and the use of stronger fibre in the core results in higher tensile strength. Typical tensile behaviours of structure-1, 2 and 3 are shown in the Fig. 10a, b and c, respectively.

The results show that the tensile behaviour of structure- 2 is completely different from structure-1 and structure-3. Even straight longitudinal rods are incorporated in structure-2, it shows lower tensile strength as compared to the basic structure due to uneven distribution of loads between the straight and diagonal longitudinal elements. Load is first taken by the straight rods when the diagonal rods start to become straight. By the time the diagonal rods become fully straight, the straight rods get fully extended and break, suddenly transferring the load to the diagonal rods, leading to their extension and breakage.

As these two types of longitudinal elements do not work together, structure-2 possess inferior mechanical properties than other structures. On the contrary, in case of structure-3, it is possible to enhance the tensile load bearing capacity by adding straight elements and also increasing diagonal rod angle. In this case, the diagonal rods take up load quickly much before the complete extension of straight rods and an even sharing of loads occurs by these two elements. As these two types of rods work together in structure-3, the tensile strength of structure-3 is significantly higher than the other two types of structures. In addition, as the diagonal longitudinal rods first straighten and then extend, the lower is the angle, the more will be the straightening action causing damage and reducing subsequent load bearing capacity of the longitudinal elements. At higher angle, there is less possibility of damage during this period, resulting in higher tensile strength of structures. This may be another reason for higher tensile properties of structure-3.

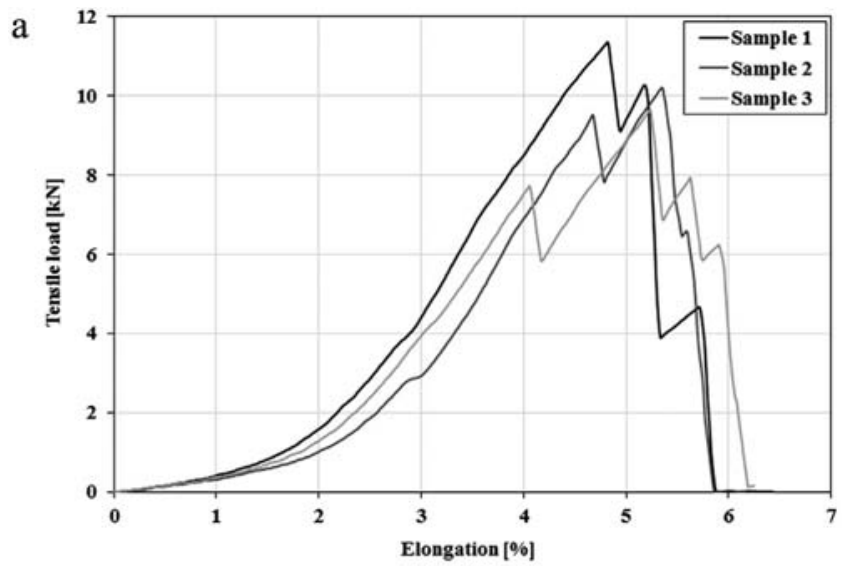

b
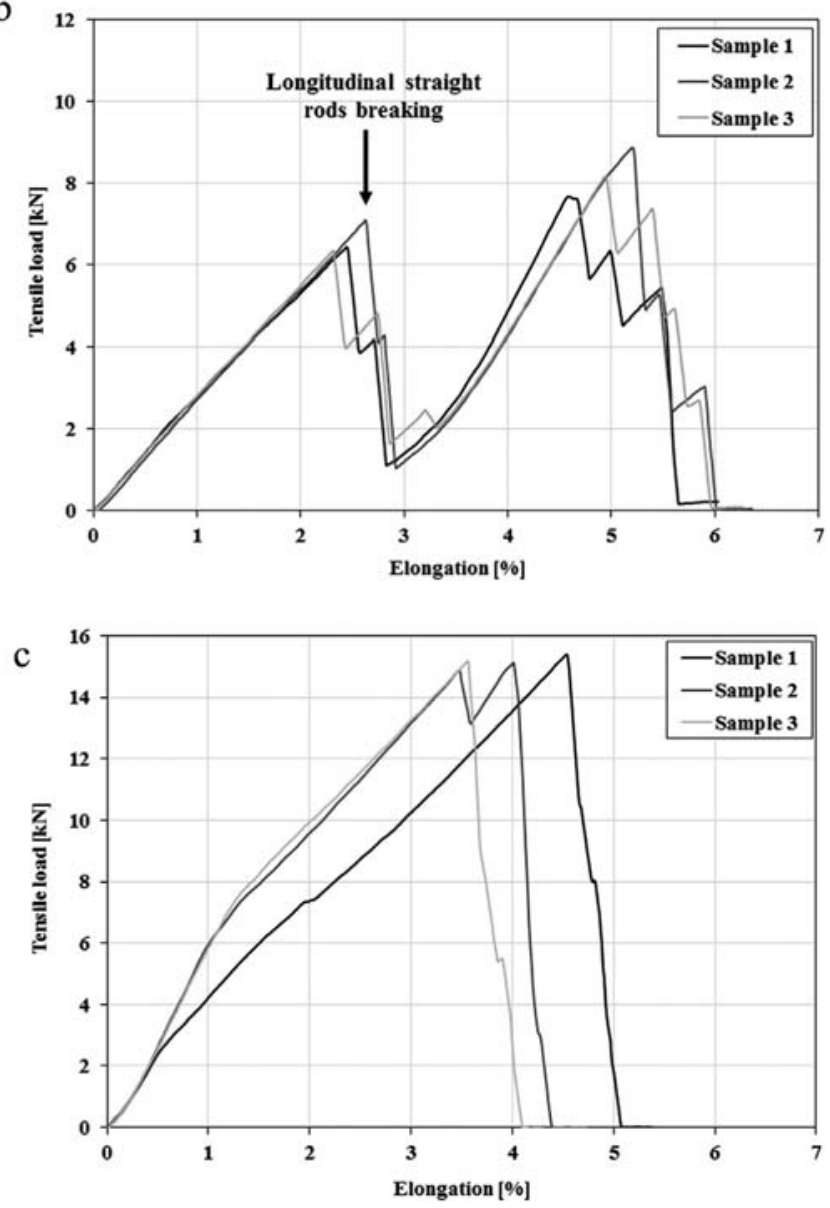

Fig. 10. a) Tensile behaviour of structure- 1 made of glass fibre BCRs, b) Tensile behaviour of structure-2 made of glass fibre BCRs, c) Tensile behaviour of structure-3 made of glass fibre BCRs. 


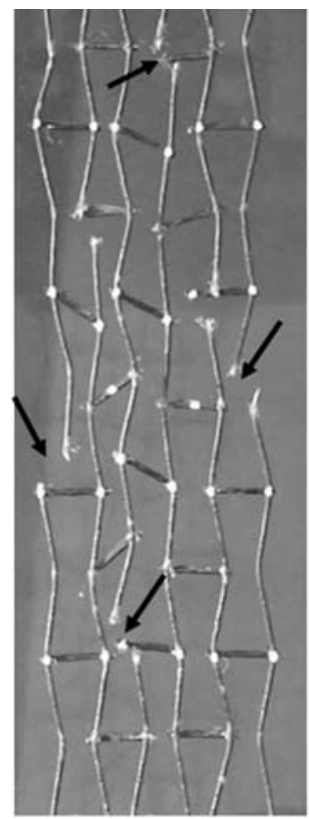

(a)

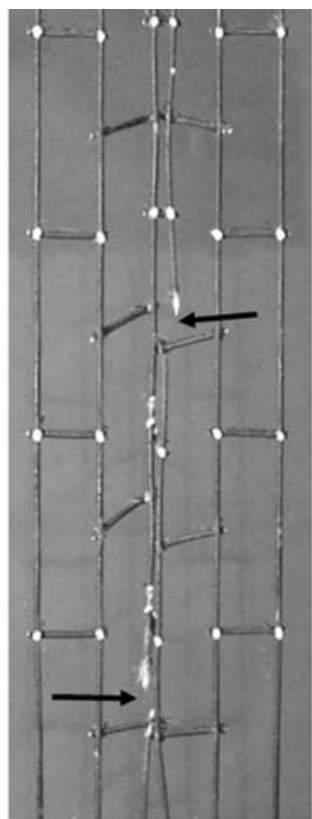

(b)

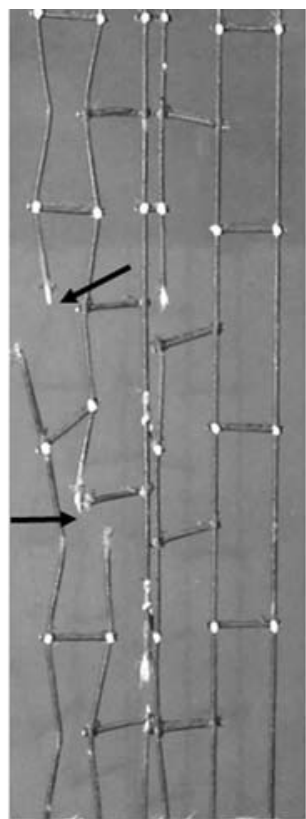

(c)

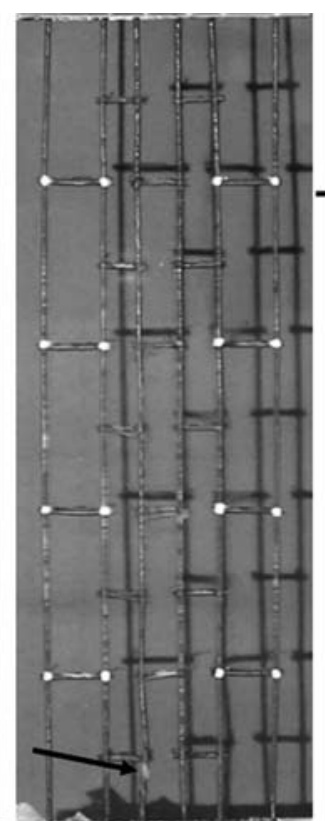

(d)

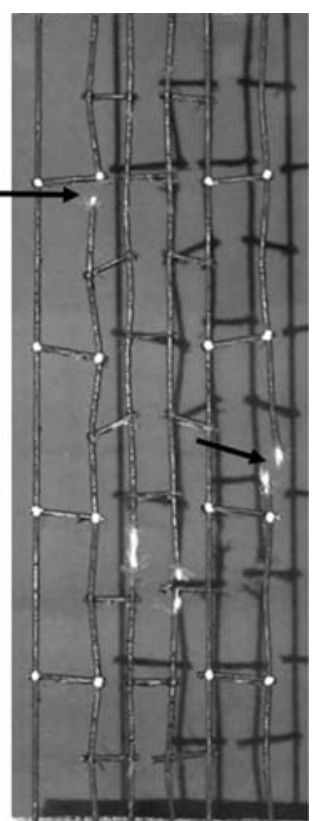

(e)

Fig. 11. Failure mode of developed auxetic structures. a) Breakage of bent rods in structure-1, b) breakage of straight rods in structure-2, c) breakage of bent rods in structure-2, d) breakage of straight rods in structure-3, and e) breakage of bent rods in structure-3.

\subsection{Failure mode of auxetic structures}

The failure modes of the developed auxetic structures are shown in Fig. 11. In these auxetic structures the linking points or ribs bases are the weak points and thus failure occurred in these locations due to stress concentration (shown by arrows in Fig. 11a). In the modified structures (structure-2,3), the failure first occurred in the straight rods as they are subjected to much higher stresses as compared to the bent rods (shown by arrows in Fig. 11b, d). Once the straight elements were broken, load was fully transferred to the bent elements leading to their straightening and finally breakage at their weak locations (shown by arrows in Fig. 11c and e).

\subsection{Work of rupture of auxetic structures}

Work of rupture (WOR) or energy required to break the structures has been calculated from the area under the load-elongation curve and presented in Table 6. Similar to tensile strength, work of rupture also increases with increase in linear density of glass fibre in case of structure-1 and is higher for higher strength core fibres, i.e. $\mathrm{WOR}_{\text {glass }}<\mathrm{WOR}$ basalt $<$ WOR carbon. Likewise, work of rupture for different structures also follows the same trend as the tensile strength, i.e. WOR structure-2 $<$ WOR structure- $1<$ WOR structure-3. As structure-3 exhibits the highest work of rupture and tensile property among these three structures, it is recommended for structural applications. Incorporation of straight longitudinal rods to the basic auxetic design and selection of a proper structural angle leads to good balance between auxetic and mechanical behaviours.

\section{Conclusions}

In this research, novel auxetic structures at macro-scale was developed using braided composite rods and the influence of various structural and material parameters on both auxetic and tensile properties was thoroughly studied and compared with analytical models. Following major conclusions can be drawn from the present research:
The developed auxetic structures exhibit negative Poisson's ratio with maximum value of -9.00 . Poisson's ratio of these structures strongly depends on the structural parameters such as structural angle, and also dependent to a lesser extent on the material parameters such as core fibre type and linear density. Tensile behaviour of the auxetic structures also depends significantly on the material and structural parameters. Higher tensile strength is observed in structures developed using stronger fibres (carbon $>$ basalt $>$ glass) and with higher linear density (6000 tex $>4800$ tex $>2400$ tex). It is also possible to tailor the mechanical properties of developed structures through incorporation of additional straight longitudinal elements. The structures developed using straight longitudinal elements (structure-2), however, exhibit lower Poison's ratio and tensile properties compared to the basic structures. Further modification of structure2 by increasing angle $\theta$ (i.e. structure- 3 ) results in higher Poisson's ratio ( -5.93$)$, tensile strength as well as work of rupture. Among the different fibres, carbon based auxetic structures exhibit the highest work of rupture, followed by basalt and glass. An increase in liner density of core fibres also increases the work of rupture of the auxetic structures.

The experimental results show that structure- 3 is suitable for structural applications as it provides both suitable auxetic behaviour (negative Poison's ratio) as well as mechanical performance (higher tensile strength and work of rupture or energy absorption capability). This composite material (structure-3) can be beneficial for protecting civil structural elements (both concrete elements and masonry walls) under impact, explosion and seismic loads due to its ductile failure. Also, these structures can be designed easily with the help of the analytical model developed within this study.

\section{Acknowledgement}

The authors gratefully acknowledge the financial support for carrying out this research work from University of Minho, under the scheme of "Strategic plan of school of engineering - Agenda 2020: Multidisciplinary projects." 


\section{References}

[1] Pichandi S, Rana S, Oliveira D, Fangueiro R. Fibrous and composite materials for blast protection of structural elements - a state-of-the-art review. J Reinf Plast Compos 2013:32(19):1477-500.

[2] Awad ZK, Aravinthan T, Zhuge Y, Gonzalez F. A review of optimization techniques used in the design of fibre composite structures for civil engineering applications. Mater Des 2012;33:534-44.

[3] Olivito RS, Cevallos OA, Carrozzini A. Development of durable cementitious composites using sisal and flax fabrics for reinforcement of masonry structures. Mater Des 2014;57:258-68.

[4] $\mathrm{Gu} \mathrm{H}$, Zhonge Z. Compressive behaviour of concrete cylinders reinforced by glass and polyester filaments. Mater Des 2005;26:450-3.

[5] Buchan PA, Chen JF. Blast resistance of FRP composites and polymer strengthened concrete and masonry structures - a state-of-the-art review. Compos Part B Eng 2009:38:509-22.

[6] Pereira CG, Fangueiro R, Jalali S, Marques PP, Araujo M. Braided composite rods to reinforce concrete subjected to aggressive environments. In: International conference construction heritage in coastal and marine environments; 2008. p. 1. Lisbon, Portugal.

[7] Ahmadi MS, Johari MS, Sadighi M, Esfandeh M. An experimental study on mechanical properties of GFRP braid-pultruded composite rods. EXPRESS Polym Lett 2009;3(9):560-8.

[8] Pereira CG, Fangueiro R, Jalali S, Marques PP, Araujo M. Hybrid composite rods for concrete reinforcement. Struct Arch Cruz (Ed) 2010:1605-12.

[9] Rana S, Zdraveva E, Pereira C, Fangueiro R, Correia AG. Development of hybrid braided composite rods for reinforcement and health monitoring structures. Sci World Jobs 2014:1-9.

[10] Rosada KP, Rana S, Pereira C, Fangueiro R. Self-sensing hybrid composite rod with braided reinforcement for structural health monitoring. Mater Sci Forum 2013;730-732:379-84.

[11] Cunha F, Rana S, Fangueiro R, Vasconcelos G. Excellent bonding behaviour of novel surface-tailored fibre composite rods with cementitious matrix. B Mater Sci 2014:37(5):1013-7.

[12] Fangueiro R, Rana S, Correia AG. Braided composite rods: innovative fibrous materials for geotechnical applications. Geomech Eng 2013;5(2):87-97.

[13] Shokrieh MM, Assadi A. Determination of maximum negative Poisson's ratio for laminate fibre composites. Phys Status Solidi B 2011;248(5):1237-41.

[14] Strek T, Jopek H. Effective mechanical properties of concentric cylindrical composites with auxetic phase. Phys Status Solidi B 2012:1-7.

[15] Donoghue JP, Alderson KL, Evans KE. The fracture toughness of composite laminates with a negative Poisson's ratio. Phys Status Solidi B 2009;9:2011-7.

[16] Alderson A, Alderson KL. Auxetic materials. J Aerosp Eng 2007;221:565-75.

[17] Yang W, Li ZM, Shi W, Xie BH, Yang MB. Review on auxetic materials. J Mater Sci 2004:39:3269-79.
[18] Prawoto Y. Seeing auxetic materials from the mechanics point of view: a structural review on the negative Poisson's ratio. Compost Mater Sci 2012;58: $140-5$.

[19] Yao YT, Uzun M, Patel I. Working of auxetic nanomaterials. J Achiev Mater Manuf Eng 2011;49(2):585-93.

[20] Miller W, Ren Z, Smith CW, Evans KE. A negative Poisson's ratio carbon fibre composite using a negative Poisson's ratio yarn reinforcement. Compos Sci Technol 2012;72:761-6.

[21] Jayanty S, Crowe J, Berhan L. Auxetic fibre networks and their composites. Phys Status Solidi B 2011;248(1):73-81.

[22] Grima JN, Caruana-Gauci R, Attard D, Gatt R. Three-dimensional cellular structures with negative Poisson's ratio and negative compressibility properties. P Roy Soc A 2013:1-18.

[23] Sloan MR, Wright JR, Evans KE. The helical auxetic yarn - a novel structure for composites and textiles; geometry, manufacture and mechanical properties. Mech Mater 2011;43:476-86.

24] Smith CW, Grima JN, Evans KE. A novel mechanism for generating auxetic behaviour in reticulated foams: missing rib foam model. Acta Mater 2008;48: 4349-56.

[25] Gaspar N, Ren XJ, Smith CW, Grima JN, Evans KE. Novels honeycombs with auxetic behaviour. Acta Mater 2005:53:2439-45.

[26] Evans KE, Anderson KL. Auxetic materials: the positive side of being negative. Eng Sci Educ J 2000:148-54.

[27] Scarpa F. Auxetic materials for bioprostheses. IEEE signal Process Mag 2008;25(5): 128-126.

[28] Hu H, Wang Z, Liu S. Development of auxetic fabrics using flat knitting technology. Text Res J 2011;81(14):1493-502.

[29] White L. Auxetic foam set for use in smart filters and wound dressings. Urethanes Technol Int 2009;26(4):34-6.

[30] Alderson A, Rasburn J, Evans KE, Grima JN. Auxetic polymeric filters display enhanced de-fouling and pressure- compensation properties. Membr Technol 2001;137:6-8.

[31] Ugbolue SC, Kim YK, Warner SB, Fan Q Yang CL, Kyzymchuk O, et al. Engineered warp knit auxetic fabrics. J Text Sci Eng 2012;2(1):1-8.

[32] Hu H, Liu Y. A review on auxetic structures and polymeric materials. Sci Res Essays 2010;5(10):1052-63.

[33] Karnessis N, Burriesci G. Uniaxial and buckling mechanical response of auxetic cellular tubes. Smart Mater Struct 2013;22:1-9.

[34] Hou Y, Neville R, Scarpa F, Remillat C, Gu B, Ruzzene M. Graded conventionalauxetic Kirigami sandwich structures: flatwise compression and edgewise loading. Compos Part B Eng 2014;59:33-42.

[35] Silva TAA, Panzera TH, Brandão LC, Lauro CH, Boba K, Scarpa F. Preliminary investigations on auxetic structures based on recycled rubber. Phys Status Solidi B 2012;249(7):1353-8.

[36] Subramani P, Rana S, Oliveira DV, Fangueiro R, Xavier J. Development of novel auxetic structures based on braided composites. Mater Des 2014:61:286-95. 